\title{
Bilateral empyema and purulent pericarditis due to Haemophilus influenzae capsular type b
}

\author{
RICHARD IGGO, ROBERT HIGGINS \\ From the Department of Cardiology, John Radcliffe Hospital, Oxford
}

ABSTRACT A previously fit woman developed a sore throat followed by bilateral empyema and pericarditis due to Haemophilus influenzae capsular type b. She was treated successfully with antibiotics, bilateral thoracotomies, and pericardotomy.

A previously fit 64 year old woman presented with a one week history of a severe sore throat. The day before admission she developed dyspnoea and right sided pleuritic chest pain. She had never smoked and took little alcohol. On examination the jugular venous pressure was not raised and in the chest there were signs of a right sided pleural effusion. Aspiration of this disclosed frank pus, and cultures of blood and pleural fluid grew Haemophilus influenzae capsular type b, sensitive to ampicillin, cefuroxime, and chloramphenicol.

She improved with insertion of an intercostal tube drain and intravenous cefuroxime ( $750 \mathrm{mg}$ eight hourly) but two days later deteriorated rapidly. The jugular venous pressure became raised, a chest radiograph showed bilateral pleural effusions and an increase in cardiac size, and two dimensional echocardiography showed a pericardial effusion. Bilateral full thoracotomies and pericardiotomy were immediately performed with aspiration of $500 \mathrm{ml}$ of pus from the pericardium and $300 \mathrm{ml}$ from the left pleural space. Biopsy of the pericardium showed only fibropurulent pericarditis. Intravenous chloramphenicol ( $500 \mathrm{mg}$ six hourly) was given until the 11 th postoperative day, when a four week course of oral amoxycillin ( $250 \mathrm{mg}$ eight hourly) was started.

On review five months later she was symptomatically well, but there was clinical evidence of mild pericardial constriction with a raised jugular venous pressure, tachycardia, and a pericardial knock. Review of her immunological state showed no defect in antibody production in response to tetanus toxoid or to pneumococcal vaccine and no evidence of underlying myeloma or lymphoma.

\section{Discussion}

This previously fit woman who developed overwhelming infection with Haemophilus influenzae type b represents, so far as we are aware, the first published case of empyema and pericarditis due to this organism occurring in an adult in this country.

Address for reprint requests: Dr R Higgins, Nuffield Department of Surgery, John Radcliffe Hospital, Oxford OX3 9DU.

Accepted 15 April 1988
Haemophilus influenzae capsular type b, unlike th non-capsulated forms that commonly cause bronchitis characteristically causes a bacteraemic illness, with dissemine ation to multiple sites.' It is rarely pathogenic in adults with adequate humoral immunity, and tends to occur in thoser immunosuppressed by alcohol or cytotoxic drug $\$$ Pneumonia in adults due to this organism is becoming more common in the United States ${ }^{2}$ and has been reported in the United Kingdom. ${ }^{3}$

Although we are unaware of any previous reports of Haemophilus influenzae pericarditis occurring in adults in Britain, at least 10 cases have been published worldwide. Typically the illness begins with a severe sore throat, whicho may itself be an underdiagnosed manifestation of aduit epiglottitis due to this organism. ${ }^{10}$ If pericarditis develop there is no record of successful treatment without drainage of the pericardium. In one case pericardial drainage wa performed by a tube inserted percutaneously ${ }^{6}$; but wit bilateral empyema, as in our case, open surgery may be preferable so that the pleural spaces can be explored and full drained during one procedure.

Our case suggests that Haemophilus influenzae type $\vec{b}$ should be suspected as a possible causative organism patients with an acute overwhelming septicaemia, particularly if there is initially a severe sore throat. It is a virulent infection and, even in patients who are otherwise fit, tho infection may spread to the pleural and pericardial spaces during appropriate antibiotic treatment. Prompt diagnosis of pericarditis with two dimensional echocardiography an emergency drainage of the pericardium may be life saving Possibly infections due to this organism will become more common in the United Kingdom, as they have in the Unite States.

We would like to thank Professor P Sleight for reading the manuscript and for permission to report this case, and $\mathrm{Mr} \mathrm{N}$ Gunning and Dr N Boon, who also managed the patient ip hospital

\section{References}

1 Turk DC. The pathogenicity of Haemophilus influenzae. J Med Microbiol 1984;18:1-16.

2 Wallace RJ, Musher DM, Martin RR. Haemophilus influenza pneumonia in adults. Am J Med 1978;64:87-93.

3 Ashworth M, Ross G, Loehry C. Lobar pneumonia caused by Haemophilus influenzae type b. Br J Dis Chest 1985;79:95-7.

4 Rubin RH, Moellering RC. Clinical, microbiologic an therapeutic aspects of purulent pericarditis. Am J Med 1975;59:68-78.

5 Alsever RN, Stiver HG, Dinerman N, Dahl CR, Eickhoff TC. 
Haemophilus influenzae pericarditis and empyema with thyroiditis in an adult. JAMA 1974;230:1426-7.

6 Keifaber RW, Bach RD, McDowell JA. Nonsurgical treatment of Haemophilus influenzae pericarditis in an adult. Am Heart $J$ 1984;108:168-9.

7 Duke M, Donovan TJ. Haemophilus influenzae pericarditis with cardiac tamponade. Am J Cardiol 1973;31:778-80.

8 Sheikh MU, Lee WR, Koh DS. Serial echographic development of apparent hypertrophic cardiomyopathy with biventricular outflow obstruction: documentation in Haemophilus influenzae pericarditis during hypovolaemia. Am Heart J 1981;102: 1069-71.

9 Buckingham TA, Wilner G, Sugar SJ. Haemophilus influenzae pericarditis in adults. Arch Intern Med 1983;143:1809-10.

10 Baker AS, Eavey RD. Adult supraglottitis. $N$ Engl $J$ Med 1986;314:1185-6.

\section{Correction}

\section{Diaphragm strength in patients with recent hemidiaphragm paralysis}

In the paper by Dr CM Laroche et al (February 1988) errors occur on pages 172 and 173. In table 3 the figures in parentheses in the last two lines are SDs and should be under the $\mathrm{cm} \mathrm{H}_{2} \mathrm{O}$ not the \% predicted columns. In table 4 the mean and SD figures should each move one column to the right.

\section{Notice}

\section{J Levy scoliosis research scholarship 1988}

Applications are invited for the $\mathbf{J}$ Levy scoliosis research scholarship from physicians, surgeons, and those in allied specialties, including those in training. The scholarship is intended for the use of graduates of British medical schools while working in orthopaedics or allied subjects in the British Isles. The scholarship is worth $£ 15000$ and is to further research into scoliosis and in particular methods of early detection and prevention. The closing date for applications is 31 August 1988. Applicants should apply, stating clearly how they intend to use the scholarship and giving a curriculum vitae, to $\mathrm{Mr}$ John Dove FRCS, secretary and treasurer of the British Scoliosis Society, 31 Quarry Avenue, Hartshill, Stoke-on-Trent ST4 7EW.

\section{Book notices}

Lung Function in Children and Adolescents. A Zapletal, M Samanek, T Paul. (Pp 228; £79.60.) Basel: Karger, 1987. ISBN 3-8055-4495-2.

There are many reports on the results of lung function tests in healthy children and it would be entirely reasonable for a potential reader to ask whether there was a need for yet more normal data. I would claim that the data in this book by Zapletal and his colleagues are different from previous reports and do make a significant new contribution. The number of children and adults included is not particularly large-173. What is different is that the children were studied with virtually all the techniques available for studying pulmonary physiology in cooperative children. These include all the standard measurements on standard spirometry, flowvolume curves, helium and plethysmographic static lung volumes, lung compliance, total pulmonary and airways resistance, gas diffusion, work of breathing, and physiological deadspace. Some data are included on the work of breathing in a small group of healthy young adults. Although this book does not provide all the information needed to set up the lung function techniques considered, Dr Zapletal and his group, who have perhaps contributed more to our knowledge of normal data on children than anybody else, have provided all the data on the methods used to define the exact circumstances in which these studies were carried out. Possible sources of error are fully described, the means and $95 \%$ confidence limits calculated, and the relationships of one measurement to another explored in considerable depth. The reference section is extensive and the latter half of the book contains 284 tables giving lung function data related to height, body surface area, and static lung volumes. I am sure that all those concerned in all but the most basic lung function measurements in children will find this book an invaluable reference source.-ADM

Essentials of Thoracic Surgery. Raymond Hurt, Michael Bates. (Pp 270; £24.50.) London: Butterworth, 1986. ISBN 0407003584 .

This book of 300 pages provides a clear, comprehensive, and yet concise guide to thoracic surgery. There are introductory chapters which cover anatomy, physiology, development and congenital abnormalities. Other chapters review preoperative investigations and the techniques of endoscopy. The style of the book is didactic and certainly not all thoracic surgeons would agree with all methods of management, but such an approach produces a uniform and very readable text. There is no condition met by thoracic surgeons which is not covered in this book, which includes a chapter on subphrenic abscess. In many areas the book excels in providing clear, practical guides such as the techniques of thoracotomy and rigid oesophagoscopy. An outstanding feature is the high quality of the radiographs, which is unusual in a book of this size. It is, however, disappointing not to find clearer details of the management of chest drains and the handling of a postoperative air leak. Some discussion of methods of improving respiratory function before thoracotomy in those with marginal function would be welcome. At the end of the book there are sections on coronary artery surgery and 